\title{
STATUS OF THE DIAMOND INSERTION DEVICES
}

\author{
J. A. Clarke, N. Bliss, D. G. Clarke, F. E. Hannon, C. Hill, A. A. Muir and D. J. Scott, Daresbury \\ Laboratory, Warrington, UK. \\ V. Francis, Rutherford Appleton Laboratory, Didcot, UK.
}

\begin{abstract}
Seven insertion devices must be provided for the first DIAMOND beamlines on Day 1 of operations. A variety of devices have been selected, including four short period in-vacuum undulators, one $5 \mathrm{~m}$ conventional planar undulator, one $5 \mathrm{~m}$ helical undulator and a $3.5 \mathrm{~T}$ superconducting wiggler. Construction of several of these devices will soon commence. This paper will detail the magnetic solutions that have been adopted and also the mechanical designs, highlighting the key features that have been included.
\end{abstract}

\section{INTRODUCTION}

The DIAMOND Light Source is now in the construction phase of the project with significant progress being made over all of the major areas [1]. Funding for the first phase of the project makes provision for seven operational insertion device based beamlines. The selection of the insertion devices for these beamlines occurred approximately 12 months ago [2] and since then the designs have been developed so that now we are about to procure the first major items for these IDs. Table 1 summarises the main parameters for the first DIAMOND IDs. Note that the $\mathrm{K}$ value quoted for the helical undulator is for the circular polarization mode.

Table 1. Summary of the parameters for the first DIAMOND IDs.

\begin{tabular}{|c|c|c|c|}
\hline Name & $\begin{array}{c}\text { Period } \\
(\mathrm{mm})\end{array}$ & $\mathrm{K}_{\mathrm{Max}}$ & Length (m) \\
\hline MPW60 & 60 & 19.6 & 1.0 \\
\hline U33 & 33 & 1.63 & 4.9 \\
\hline U23 IVa & 23 & 1.49 & 2.0 \\
\hline U23 IVb & 23 & 1.49 & 2.0 \\
\hline U21 IV & 21 & 1.24 & 2.0 \\
\hline U27 IV & 27 & 2.02 & 2.0 \\
\hline HU64 & 64 & 3.44 & $2 \times 2.2$ \\
\hline
\end{tabular}

\section{IN-VACUUM UNDULATORS}

Since DIAMOND will make use of a significant number of in-vacuum undulators, with four initially but probably more than ten when the ring is fully populated, it was decided to design and build these in-house. This then ensures there is a common design philosophy and inhouse expertise available to maintain the magnets during the operational phase of the project. Although four undulators are required before operations begins it was further decided to build a single device first to ratify the design and assembly process and then to build the other three approximately 15 months later.

\section{Mechanical Design}

The mechanical design of the in-vacuum undulators is very closely based upon the ESRF design [3] although some fundamental changes have also been made. In particular, the ability to taper the magnet arrays has been added and also the rf finger taper sections at each end of the device will be water cooled. The array tapering system is based upon the rotation of offset cams at one end of the magnet array support beam (outside of the vacuum) with a pivot at the other end.

The nominal minimum beam stay clear in the central $2 \mathrm{~m}$ of the $5 \mathrm{~m}$ ID straights is set by lifetime considerations to be $7 \mathrm{~mm}$. This value has been used to design the invacuum undulators to meet the needs of the users. However, mechanically the magnets are designed to reach an absolute minimum gap of $4 \mathrm{~mm}$ so that if smaller gaps can be tolerated by the machine the users will be able to benefit. The maximum magnet gap has been selected to be $30 \mathrm{~mm}$.

There are already serious proposals for a second undulator to be installed in the same straight as an invacuum undulator to serve a side station (see Figure 1). Although the in-vacuum undulator magnet blocks fill a length of $\sim 2.0 \mathrm{~m}$ it is physically $\sim 2.6 \mathrm{~m}$ because of the room needed for the rf tapers, bellows, flanges etc. Allowing some space for steering magnets to apply an angular offset ( $1 \mathrm{mrad}$ ) between the radiation from the two undulators leaves a length of $\sim 0.8 \mathrm{~m}$ for a second (out of vacuum) undulator. It is planned to install the corrector magnets and the in-vacuum undulator with a horizontal angular offset from Day 1 to enable easier introduction of the second undulator in the future.

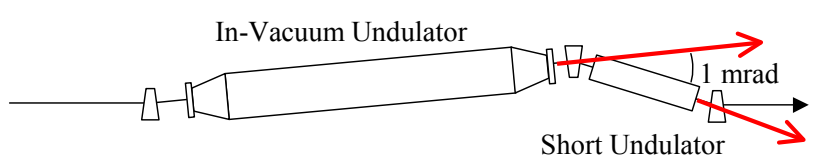

$$
\underset{\text { Figure 1. Sketch of proposed layout of DIAMOND }}{\text { straight. }}
$$

\section{Magnet Design}

The choice of magnet material for in-vacuum undulators is an interesting issue [4]. Since the magnets have to be baked to $\sim 140{ }^{\circ} \mathrm{C}$ irreversible losses will occur with $\mathrm{NdFeB}$ magnets but not with SmCo magnets. Despite these magnetization losses the $\mathrm{NdFeB}$ magnets 
will still give a larger on axis field than the intrinsically weaker SmCo. Also, since the in-vacuum undulators are often the limiting vertical aperture in a storage ring they can experience high radiation doses. There is evidence that $\mathrm{NdFeB}$ magnets are more susceptible to radiation damage than $\mathrm{SmCo}[5,6]$ and also that the $\mathrm{Sm}_{2} \mathrm{Co}_{17}$ grade is more resistant than $\mathrm{SmCo}_{5}$. For the reasons outlined above we decided to base our magnet designs on $\mathrm{Sm}_{2} \mathrm{Co}_{17}$ with an assumed remanent field of $1.03 \mathrm{~T}$.

The magnet design is a conventional symmetric pure permanent magnet four block per period structure with block height and width of 16 and $41 \mathrm{~mm}$ respectively. The end design is shown schematically in figure 2 . The length of the last block has been optimised to minimise the $1^{\text {st }}$ field integral over the full range of operating gaps. Figure 3 shows how the integral varies for different block lengths. The chosen length of $2.7 \mathrm{~mm}$ gives a theoretical maximum integral without block errors of less than $0.5 \mu \mathrm{Tm}$ which corresponds to an angular deviation of $0.05 \mu \mathrm{rad}$ for the $3 \mathrm{GeV}$ electron beam.

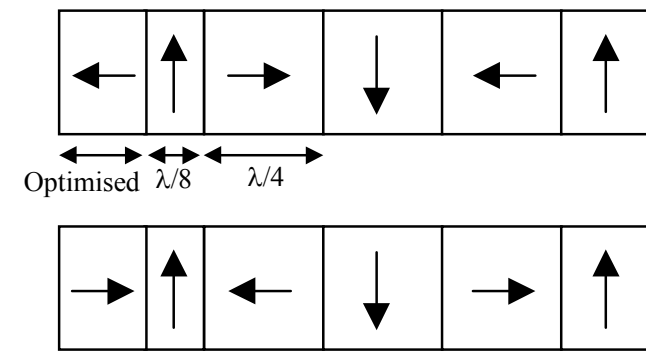

Figure 2. End structure adopted for the in-vacuum undulator.

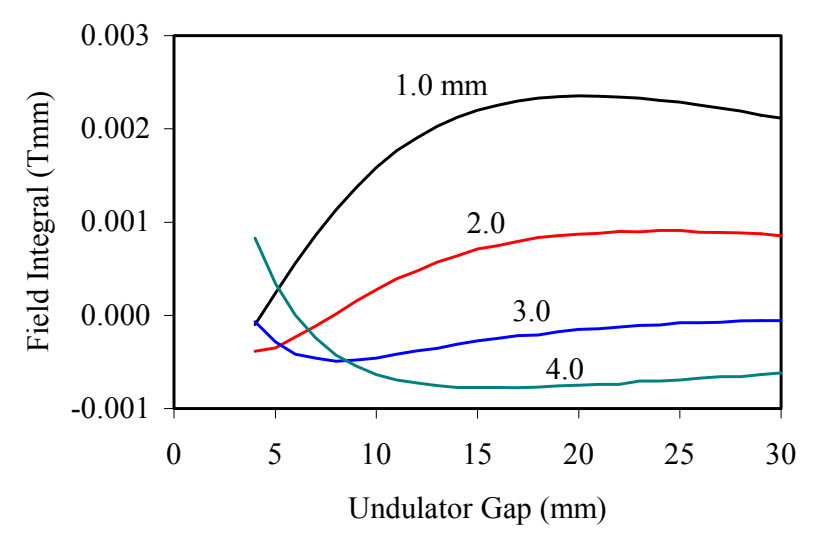

Figure 3. First field integral as a function of undulator gap for different end block lengths.

\section{HELICAL UNDULATOR}

For the same reasons outlined for the in-vacuum undulator it was decided to design and build the out of vacuum permanent magnet undulators in-house as well. A common support structure philosophy was also adopted whereby all out of vacuum undulators will use the same structure design as far as possible. It is relatively easy to alter the length of the support structures for different length modules as necessary.
It was also decided that the helical undulator, which was originally planned to be $5 \mathrm{~m}$ long built of two modules, should actually be two separate modules acting independently with an electromagnetic phasing unit placed in-between. This gives more flexibility to the beamline operation in the use of the polarization as well as easing the need for a complex interlock system to prevent one module striking another. Overall, a few periods have to be sacrificed to make space for the phasing unit but the loss in flux is not critical.

Also, the ability to taper the magnet beams has been included in the support structure design by implementing a four motor system (two per beam) for the gap changing mechanism.

The helical design adopted is the APPLE-2 configuration [7] which requires a longitudinal phasing movement of one magnet array on each support beam. However, it is possible that future designs might adopt a three array per beam system and so the phase change mechanism has been designed to cope with this. The forces generated by the undulator can be very large, particularly in the axial direction when the diagonally opposite arrays are moved in opposite directions to generate arbitrary inclination linear polarization. The phase change mechanism is designed to cope with forces up to $40 \mathrm{kN}$. The forces on each array were calculated as a function of the magnet block size (Figure 4). A block size of $40 \times 40 \times 16 \mathrm{~mm}$ was chosen as a compromise between magnet field strength and quality and the forces generated. A view of the HU64 mechanical support structure is given in Figure 5.

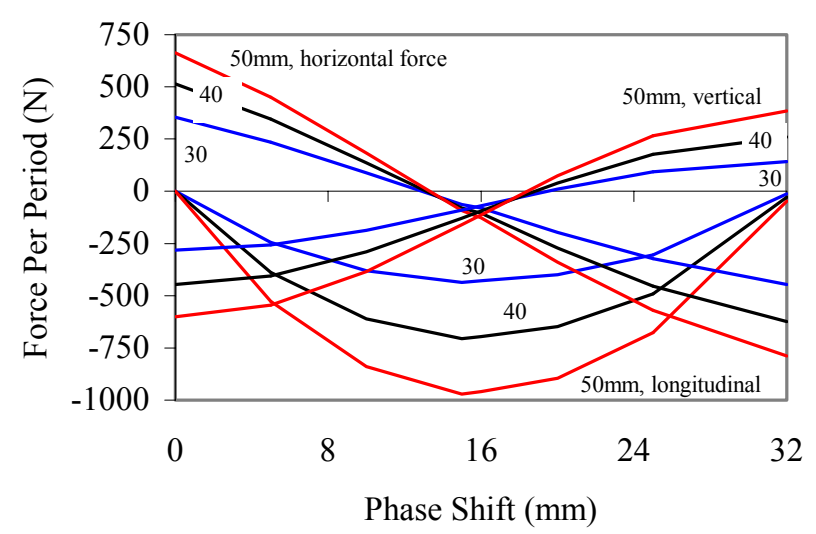

Figure 4. Force per period on the HU64 undulator operating in the arbitrary linear polarization mode at a $15 \mathrm{~mm}$ gap as a function of transverse dimension for square magnet blocks. 


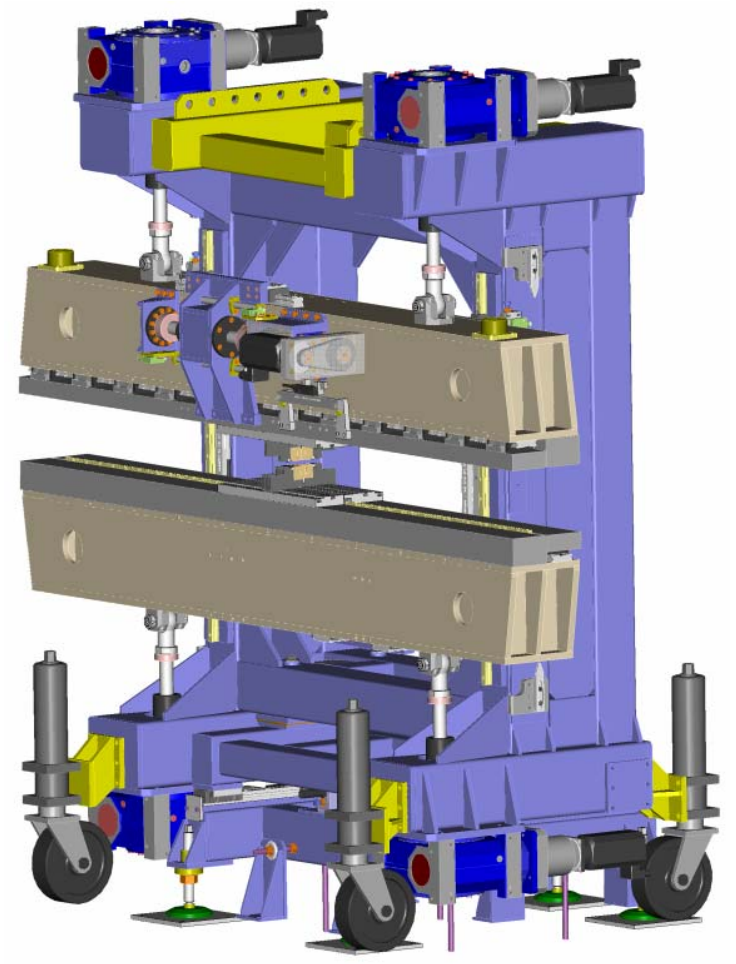

Figure 5. View of the helical undulator support structure. Only a few magnet blocks are shown mounted on the beams.

\section{PLANAR UNDULATOR}

Mechanically the $4.9 \mathrm{~m}$ long planar U33 will be very similar to the helical undulator described above, though without the phase changing mechanism of course. It will consist of two modules mounted very closely together to form one continuous undulator. The exact space between the magnets at this junction has yet to be selected.

Magnetically it is a relatively straightforward pure permanent magnet planar undulator with four blocks per period. The end structure is expected to be similar to that described for the in-vacuum undulator.

\section{SUPERCONDUCTING MULTIPOLE WIGGLER}

The superconducting multipole wiggler is the only Day 1 device that will not be built in-house. Instead a specification will be written for a turn key system that is expected to include the magnet with cryogenics, power supplies, control system etc.

As yet the exact requirements for this magnet have not been agreed with the beamline users. This is because a 3.5T MPW can produce a considerable amount of power $(\sim 21 \mathrm{~kW} / \mathrm{m}$ in DIAMOND) and the beamline designers have to be satisfied that such high power levels can be coped with. The magnet length is likely to be set between 1 and $2 \mathrm{~m}$.

\section{REFERENCES}

[1] R.P. Walker, "Progress with the DIAMOND Light Source Project", these proceedings.

[2] J.A. Clarke, F.E. Hannon and D.J. Scott, "The Initial Insertion Devices for the DIAMOND Light Source", EPAC 2002, p2607.

[3] J. Chavanne, C. Penel and P. Van Vaerenbergh, "Construction of APPLE II and In Vacuum Undulators at ESRF", PAC 2001, p2459.

[4] J. Chavanne et al, "Magnetic Design Considerations for In-Vacuum Undulators at ESRF", EPAC 2002, p2604.

[5] Y. Ito et al, "Magnetic Flux Loss in Rare-Earth Magnets Irradiated with $200 \mathrm{MeV}$ Protons", NIM B 183 (2001) p323.

[6] T. Ikeda and S. Okuda, "Magnetic Flux Loss of the Permanent Magnets used for the Wigglers of FELs by the Irradiation with High-Energy Electrons or XRays", NIM A 407 (1998) p439.

[7] S. Sasaki, "Analyses for a Planar Variably Polarizing Undulator”, NIM A 347 (1994) p83. 